\title{
Coulisses
}

Revue de théâtre

\section{Un personnage plus faustien que Faust}

Entretien avec le dramaturge Ewald Palmetshofer. Propos recueillis et traduits par Catherine Mazellier-Lajarrige

\section{Ewald Palmetshofer et Catherine Mazellier-Lajarrige}

Traducteur : Catherine Mazellier-Lajarrige

\section{OpenEdition \\ Journals}

Édition électronique

URL : https://journals.openedition.org/coulisses/213

DOI : 10.4000/coulisses. 213

ISSN : 2546-9460

\section{Éditeur}

Presses universitaires de Franche-Comté

\section{Édition imprimée}

Date de publication : 31 décembre 2011

Pagination : 17-22

ISBN : 978-2-84867-404-9

ISSN : $1150-594 \mathrm{X}$

\section{Référence électronique}

Ewald Palmetshofer et Catherine Mazellier-Lajarrige, " Un personnage plus faustien que Faust », Coulisses [En ligne], 43 | Automne 2011, mis en ligne le 30 novembre 2016, consulté le 29 décembre 2022. URL : http://journals.openedition.org/coulisses/213 ; DOI : https://doi.org/10.4000/coulisses 213 


\section{Un personnage plus faustien que Faust}

Entretien avec le dramaturge Ewald Palmetshofer. Propos recueillis et traduits par Catherine Mazellier-Lajarrige

Ewald Palmetshofer et Catherine Mazellier-Lajarrige

Traduction : Catherine Mazellier-Lajarrige

\section{NOTE DE L'ÉDITEUR}

L'original a paru dans la postface de : Ewald Palmetshofer, faust hat hunger und verschluckt sich an einer grete / faust a faim. immangeable marguerite, trad. Laurent Muhleisen, Catherine Mazellier et Hilda Inderwildi, Toulouse, Presses Universitaires du Mirail, « Nouvelles Scènes. Allemand », 2011, p. 195-200. En accord avec l'auteur, l'usage des minuscules pour désigner « faust » et « grete » n'est conservé que dans le titre de la pièce.

Cet entretien a eu lieu le 10 mars 2011 à Vienne. Ces propos ont été recueillis et traduits de l'allemand par Catherine Mazellier-Lajarrige.

CATHerine mazellier - Ewald Palmetshofer, quelle importance accorder à l'opposition entre la ville et la campagne, entre la grande ville et la province dans votre pièce faust a faim. immangeable marguerite?

Ewald PALMETSHOFER - Ce n'est pas tellement le déséquilibre géographique entre grand centre urbain et périphérie qui m'importe. L'antithèse ville / campagne, je l'associe avant tout à une différence dans l'accès aux ressources et à l'éducation. En même temps, j'ai le sentiment que la ruralité a une autre connotation que la précarité urbaine. Il ne s'agit pas simplement d'une structure binaire, mais du fait que, dans le regard des citadins, les régions rurales sont encore chargées d'un fort romantisme social. Bien sûr, la ruralité est toujours perçue comme une chose abîmée et détruite, et on la croit parfaitement capable d'une régression à un stade d'avant la civilisation, mais dans le fond, tout cela peut toujours être neutralisé par la nature, par le 
paysage. En réalité, la province n'est jamais une menace pour les grands centres urbains.

Catherine MAZELLIER - Cet aspect est-il présent à travers le personnage de Grete?

Ewald PALMETSHOFER - Oui et non. Il s'exprime de façon peut-être plus radicale dans ma dernière pièce [tier. man wird doch bitte unterschicht / animal. on glisse tout de même vers les couches inférieures, création au Staatsschauspiel de Dresde le 11.09.2010]. Elle aborde beaucoup plus nettement le danger que représentent les régions rurales et le regard quasiment sociologique qui passe à côté de cet aspect. Dans cette pièce, si le personnage principal féminin ne cesse d'avoir le mot «animal » à la bouche, c'est pour évoquer non pas la violence originelle ou bien un potentiel primitif, mais ce qui est soustrait à l'être humain, ce qui est bafoué, comme un chien battu. Ici, l'animal n'est pas le loup, il ne symbolise pas le danger du non-apprivoisé, c'est le chien battu, par opposition au loup. C'est l'être humain aliéné qui dit «je suis un animal », non pas une bête sauvage, mais la part restante a perdu son humanité. Dans faust a faim, Grete porte certes ce danger en elle, mais en fin de compte, elle le retourne contre elle-même. Pour le groupe, elle n'est dangereuse que tant que subsiste l'incertitude sur l'enfant mort retrouvé dans la forêt : à savoir s'il s'agit d'Ismaël ou de son propre enfant.

Catherine MAZELLIER - Et l'incertitude est maintenue à dessein ?

Ewald PALMETSHOFER - Oui, provisoirement. Bien sûr, il est probable qu'il s'agisse de l'enfant de Grete, beaucoup d'éléments parlent en faveur de cette hypothèse. En même temps, jusqu'à la fin de la pièce, un tout autre scénario reste possible. Lorsqu'Anne et Fritz parlent du babyphone dans la dernière scène, il est possible que cet objet revête soudain une autre signification que pendant les scènes de fête, au début de la pièce. Tout à la fin, il se pourrait que ce babyphone soit la trace de l'enfant mort, un reste de normalité, la tentative de ce couple orphelin de ne pas laisser entrer dans sa vie la mort de l'enfant. On se passe le babyphone même si plus aucun son n'en sort. L'allusion au danger que représente Grete est donc contenue dans l'éventualité de l'autre enfant mort. Mais finalement, Grete s'en prend à ellemême et probablement à son propre enfant, en tout cas elle porte atteinte à son corps. Sa dangerosité, elle-même n'y a pas accès, c'est plutôt une faiblesse, une insatisfaction, un manque, et non pas une ressource dont elle pourrait tirer parti.

Catherine MAZELLIER - Vous portez un regard analytique critique sur les relations humaines, en particulier sur le couple. Qu'est-ce qui motive cette analyse presque structuraliste?

Ewald PALMETSHOFER - Ce qui m'a poussé à écrire cette pièce, c'était la question de notre conception du bonheur. Je crois que notre notion du bonheur est profondément individualiste. Nous ne pouvons plus penser cette notion autrement que comme bonheur de l'individu, et le seul pluriel qui soit encore envisageable, c'est l'individu redoublé en tant que couple. Nous sommes démunis face à une notion collective et politique du bonheur, qui se demande si le bonheur ou le malheur des autres pourrait encore bouleverser le cours de notre vie.

Catherine MAZELLIER - L'engagement humanitaire et le personnage de l'assistance sociale dans votre pièce expriment-ils une possible notion de bonheur collectif?

Ewald PALMETSHOFER - Il ne s'agit que d'une intervention, pas de politique. Pour reprendre la différence entre police et politique, l'engagement humanitaire relève encore de la police, et l'assistance sociale aussi. Cela ne change rien au fait que notre 
discours sur le bonheur ne fait pas de place aux autres. Je crois que c'est l'aboutissement d'une longue histoire, une histoire de la tradition chrétienne. La question, c'est justement de savoir ce que signifie Faust aujourd'hui et ce qu'est ce «bel instant» dans un monde sans Dieu, post-religieux, où l'on n'est plus sous la protection d'un contre-monde transcendant.

Catherine mazellier - Mais ce n'était déjà plus le cas pour le Faust de Goethe.

Ewald PALMETSHOFER - Oui, pour Goethe non plus, je crois. Sa pièce porte un regard ironique sur le monde, mais la réception n'en a pas toujours tenu compte. Car c'est un scandale, ce Dieu du prologue, une représentation terrible. La morale, "L'homme erre aussi longtemps qu'il cherche ", montre un Dieu qui ne se laisse plus perturber par rien, cette phrase rend toutes choses égales. Dans mon faust, la seule chose qui se prête à l'universalisation, c'est la morte dans la forêt. Seule l'image de la cabane dans la forêt, seul l'enfant mort peuvent dire le «bel instant " qui soit compréhensible partout, c'est-à-dire en fait le malheur radical. Bien sûr, cela prend une forme exacerbée, mais il faut bien faire le triste constat que nous n'avons pas de politique du bonheur pour le grand nombre, qui se laisserait perturber par le malheur là où il se trouve. Toute la pièce tourne autour de cela. C'est ce qui sert de substrat à ce groupe. Car au fond, Grete n'existe pas, seul le groupe existe.

Catherine MAzeLLIer - Et y a-t-il un Méphisto?

Ewald PALMETSHOFER - Je crois que Méphisto, c'est le groupe tout entier. Il séduit, dirige le désir d'Henri, le conduit vers un objet. Il a le côté entremetteur de Marthe et de Méphisto et tente de persuader Henri de rester auprès de cet objet.

Catherine mazellier - Chez Goethe, Méphisto soumet Henri à la tentation purement charnelle, celle de la jouissance et de la possession. Comment se comporte le groupe chez vous?

Ewald PALMETSHOFER - Je ne crois pas qu'il y ait cette séparation entre la chair et le sentiment, ou l'âme, par exemple quand Paul tente de persuader Henri qu'il faut tout de même faire des efforts pour être heureux. Il ne s'agit pas tout simplement de mettre Grete dans son lit. Ce qu'il lui conseille, c'est la totalité des possibilités. Du sexe, ils peuvent tous en avoir d'une manière ou d'une autre, ce n'est pas un souci. Ce n'est pas une aspiration simplement charnelle. Les temps ont changé. Pour moi, il était important de se demander comment raconter aujourd'hui cette Grete. Elle ne peut plus être une jeune fille de seize ans qui ne sait pas du tout comment ce rapport sexuel lui arrive. C'est une femme adulte, la trentaine dépassée, et ce n'est pas une victime du désir masculin. En fait, c'est elle le personnage le plus radicalement faustien, parce que c'est elle qui va jusqu'au bout de son désir, celle qui dit : "S'il n'est pas possible de tout avoir, alors mieux vaut ne rien avoir du tout ». Elle est face à cet absolu comme si c'était sa dernière chance : c'est maintenant ou jamais. Et en cela, c'est un personnage beaucoup plus faustien que Faust. Il m'importait de l'arracher à cette image de victime, avec des nattes blondes et ce côté « ah, je ris de me voir si belle en ce miroir ». Et à la fin, il n'y a pas de voix qui descende du ciel pour dire : «Elle est sauvée ». Cette voix n'existe pas. C'est la mort et l'échec dans toute leur radicalité. 
Catherine MAZELLIER - Au fond, Faust savait déjà dans la version primitive, le Urfaust, qu'il n'y a pas de «salut » à la fin, c'est un élément ajouté plus tard, dans la dernière phase de création, en guise de consolation.

Ewald PALMETSHOFER - Et ce revirement est impossible aujourd'hui. Croire qu'à la fin un deus ex machina va venir et que tout finira bien, cela ne marche plus. Les morts sont irrémédiablement morts et c'est le dernier mot. Ce qui est définitif n'est pas annulé. D'où aussi le fait que les personnages parlent beaucoup à la fin de la pièce. Parce qu'il n'y a pas de voix pour prodiguer des paroles d'en haut.

Catherine MAZELLIER - Pour en revenir à la question du bonheur individuel dans votre pièce, la quête d'un bonheur à deux semble se résumer à une restriction de la liberté. N'existe-t-il pas de modèle alternatif?

Ewald PALMETSHOFER - Ce qui m'importe, ce n'est pas du tout le regard porté sur le fonctionnement interne de la relation, un éventuel sentiment de restriction, mais c'est le couple en public : la façon dont les couples se donnent à voir, à quel point on se ferme au monde, à la société, pour devenir un tout organique, pour n'être qu'un, et non pas deux.

Catherine MAZELLIER - Peut-on y voir précisément la façon dont vous concevez la mission du théâtre, par opposition à une approche psychologique?

Ewald PALMETSHOFER - La dynamique psychologique de la relation ne m'intéresse pas particulièrement. Pour moi, c'est la dimension politique de la relation qui est intéressante. Pourquoi nous fuyons nos responsabilités en nous réfugiant dans la relation, telle est la question. La relation bourgeoise est-elle ce qui nous fait miroiter une diminution de la menace que représente le monde extérieur? Ce bonheur du « deux ne faisant qu'un » est-il l'espoir suprême parce que nous échouons dans notre confrontation avec le monde, parce que nous avons perdu tout espoir de changer la société?

Catherine MAZELLIER - Vous rejetez ainsi l'idée d'un théâtre psychologique?

Ewald PALMETSHOFER - La psychologie au théâtre entraîne toujours un trop-plein de causalité, elle n'est jamais surprise par une révolution parce qu'elle croit toujours comprendre après-coup. Cependant, il y a des choses que nous faisons sans les comprendre. Il y a des changements que l'on pourra éventuellement expliquer a posteriori mais qui, avant le passage à l'acte, ne semblent pas si nécessaires. La psychologie peut toujours transformer le discontinu de l'être humain en lignes de continuité. Cela rend les actions plates parce qu'on leur enlève ainsi de leur tranchant.

Catherine MAZELLIER - La langue est-elle là pour montrer le tranchant ? Je songe par exemple aux ellipses dans votre écriture : ont-elles une signification sociale?

Ewald PALMETSHOFER - Je crois que cette langue ne doit pas être interprétée sous un angle psychologique, par exemple dans le sens d'une incommunicabilité. Il ne s'agit pas de représenter une intériorité au moyen de la langue mais de rendre visible un processus de positionnement permanent. C'est intentionnellement que les phrases ne s'achèvent pas sur des points de suspension. Elles ne s'écoulent pas comme des gouttes de pluie. Les personnages ne cessent de recommencer du début, chaque phrase est une nouvelle tentative parce que cette intériorité n'existe pas et que l'on a toujours le sentiment de ne pas être exact ou suffisamment précis. Ou bien on se surprend à dire certaines choses. 
Catherine mazellier - Cette intériorité, justement, se défınit-elle par l'image que l'on donne à l'extérieur?

Ewald PALMETSHOFER - Oui, et avant même que l'image ne soit présente, il faut déjà passer à la suite, parce que la langue n'est pas en mesure d'exprimer la substance, le noyau. Dans le meilleur des cas, il se constitue au fil de la pièce quelque chose qui désigne ce noyau en creux. Cela émerge précisément au fil du déroulement.

Catherine MAZELLIER - Votre travail avec les acteurs pour les mises en scène de Mannheim et de Vienne allait-il dans cette direction?

Ewald PALMETSHOFER - Je crois que les acteurs et actrices connaissaient déjà ce processus par ma pièce hamlet est mort ${ }^{1}$. On se rend compte pendant les répétitions que les phrases ne peuvent recevoir un contenu psychologique. Sinon, vous êtes éjecté. Il faut un rythme, une vitesse intérieure.

Catherine mAZELLIER - Le fait que les rôles de Grete et Henri soient répartis sur plusieurs acteurs va aussi à l'encontre de la psychologie.

Ewald PALMETSHOFER - Cela empêche l'identification. C'est une frustration : chaque fois que l'on a le sentiment de "posséder » le personnage de Grete, il y en a une autre qui vient pour vous l'enlever et continuer à jouer Grete. Le personnage circule à travers les corps.

Catherine MAZELLIER - Un choix dramaturgique, donc.

Ewald PALMETSHOFER - La question était: comment jouer la mort sur scène ? Je ne voulais pas d'une Grete qui meure sur scène et vienne saluer à la fin. Si l'on prend la mort au sérieux, il s'agit de la faire ressentir comme un fait irrémédiable, comme le manque d'un être. On ne peut donc pas jouer à mourir, au contraire, le personnage doit être absent dès l'origine. Nous avons six personnages sur scène, et à un moment, on a le sentiment qu'il y en sept, puis huit. Mais il y a cette femme morte qui manque.

\section{NOTES}

1. hamlet ist tot. keine schwerkraft : création en novembre 2007 au Schauspielhaus de Vienne, mise en scène : Felicitas Brucker (NdT). 
INDEX

oeuvrecitee Faust, faust hat hunger und verschluckt sich an einer grete / faust a faim.

immangeable marguerite

Keywords : intertextuality

Palavras-chave : intertextualidade

Mots-clés : réécriture

Palabras claves : reescritura

\section{AUTEURS}

\section{EWALD PALMETSHOFER}

Dramaturge, écrivain

CATHERINE MAZELLIER-LAJARRIGE

Université de Toulouse II-Le mirail, CREG 